\title{
Serum levels of the high-mobility group box 1 protein (HMGB1) in children with type 1 diabetes mellitus: case-control study
}

\author{
IGOR MARJANAC $C^{1,2}$, ROBERT LOVRIĆ, JERKO BARBIĆC, \\ ${ }^{1}$ Paediatric Clinic, Osijek University Hospital, Osijek, Croatia \\ ${ }^{2}$ Department of Paediatrics, Faculty of Medicine, J. J. Strossmayer University of Osijek, Croatia \\ ${ }^{3}$ Department of Nursing and Palliative Medicine, Faculty of Dental Medicine and Health, J. J. Strossmayer University of Osijek, Croatia \\ ${ }^{4}$ Department of Nephrology, Osijek University Hospital, Osijek, Croatia \\ ${ }^{5}$ Department of Pathophysiology, Faculty of Medicine, J. J. Strossmayer University of Osijek, Croatia
}

\begin{abstract}
Introduction: The involvement of the high-mobility group box 1 protein (HMGB1) in various autoimmune and inflammatory diseases has been documented; however, the role of this proinflammatory molecule in children with diabetes type 1 (T1DM) has not been addressed. The aim of this case-control study is to compare the serum level of HMGB1 in children with newly diagnosed T1DM (group 1) and a control group composed of healthy children.

Material and methods: This case-control study included 136 children: group $1(n=96)$ and a control group $(n=40)$. Measurements were taken from serum for the following: HMGB1, white blood cell count, $C$-reactive protein, glucose, haemoglobin $A_{1 C}$ and $\beta$-cell autoantibodies (GADA-65, IA-2, ICA). HMGB1 was determined using enzyme-linked immunosorbent assay on a Labsystems iEMS Reader MF analyser (Labsystems Diagnostics Oy, Helsinki, Finland).

Results: The level (median and interquartile range) of HMGB1 was statistically higher $(p<0.001)$ in children with T1DM: 8.7 (5.0-9.8) $\mu \mathrm{g} / \mathrm{l}$, in comparison with the control group: $1.0(0.6-1.4) \mu g / l$. No correlation was found between HMGB1 and HbAlc in group 1, or between HMGB1 and BMI. A statistically higher percentage of positive children for autoantibodies were present in group 1 compared to the control group $(p \leq 0.001)$. HMGB1 serum levels were also tested and the presence of autoantibodies, and none of those antibodies correlated with the level of HMGB1.

Conclusions: The higher level of HMGB1 in children with T1DM, compared to the control group, indicates that this proinflammatory molecule is a good candidate marker of inflammation in children with TIDM.
\end{abstract}

Key words: children, inflammation, type 1 diabetes, high-mobility group box 1 protein, alarmins.

(Centr Eur J Immunol 2019; 44 (1): 33-37)

\section{Introduction}

Several studies indicate that the development of type 1 diabetes mellitus is (T1DM) related to an autoimmune reaction directed towards the Langerhans cells, which leads to their destruction and disease manifestation [1]. Furthermore, the autoimmune inflammatory reaction plays an important role in the development of diabetic complications such as retinopathy, cardiomyopathy, stroke, kidney failure, and peripheral vascular disease [2].

Both innate and adaptive immunity are involved in the development of T1DM. The cells of innate immunity mediate initial inflammatory reaction. These cells express pattern recognition receptors (PRR) that detect conserved pathogen-associated molecular patterns (PAMP) and/or damage-associated molecular patterns (DAMP) expressed by microbes or damaged human cells, respectively. It has been shown that tissue inflammation mediated by DAMP and PAMP is important for delivering the second signals that are required for the activation of adaptive T-cell immunity, which finally leads to the destruction of the Langerhans cells and the development of T1DM [3]. Among DAMP molecules, considerable attention in recent years has been drawn to the high-mobility group box 1 protein (HMGB1). HMGB1 is a highly conserved protein present in the nucleus of almost all cell types. It plays an important role in maintaining the nucleosome structure and regulation of gene transcription. It was shown recently

Correspondence address: Prof. Jerko Barbić, Department of Pathophysiology, University JJ Strosmayer Faculty of Medicine Osijek,

4 J. Huttlera St., 31000, Osijek, Croatia, e-mail: jbarbic@mefos.hr

Submitted: 27.01.2018; Accepted: 15.03.2018 
that HMGB1 can be released into the extracellular milieu passively from necrotic, pyroptotic, and/or apoptotic cells and actively by activated inflammatory cells $[4,5]$. It is involved in inflammatory responses and tissue repair. HMGB1 interacts with multiple pro-inflammatory receptors including the receptor for advanced glycation products and TLR2/4/9 PRR [6-8]. Previous animal studies have demonstrated that HMGB1 is a potent innate immune mediator contributing to autoimmune progression during T1DM, indicating the involvement of HMGB1 in T1DM pathogenesis. HMGB1 released from the late apoptotic $\beta$ cells potently activated dendritic cells in non-obese diabetic (NOD) mice, which in turn initiated an autoimmune response against $\beta$ cells, leading to their destruction [9]. Moreover, a study showed that the neutralisation of HMGB1 by specific monoclonal antibodies in NOD mice delayed diabetes onset and prevented disease progression, giving hope for the development of novel therapeutic strategies to mitigate or prevent T1DM [10]. It was also shown that treatment with an anti-HMGB1 antibody leads to a reduction of tissue inflammation in various pathological conditions such as atherosclerosis and adiposity-related liver inflammation $[11,12]$. The role of HMGB1 in the development of late diabetic complications in adult type 2 diabetes is well documented [13, 14]. However, there are no studies investigating the role of HMGB1 in juvenile T1DM.

The aim of this case-control study is to compare the serum level of HMGB1 in children with newly diagnosed T1DM with the level in a control group comprised of healthy children, without evident inflammatory pathology (normal levels of C-reactive protein [CRP] and white blood cell [WBC] count).

\section{Material and methods}

\section{Subjects}

In this case-control study a total of 136 children aged from five to 18 years (median 14 years) were enrolled. Among these subjects, 96 were children with newly diagnosed T1DM admitted to the Paediatric Department with hyperglycaemia with or without diabetic ketoacidosis as the first manifestation of the disease (group 1, $n=96$ ). Diagnostic criteria for T1DM were based on blood glucose level : casual plasma glucose concentration $\geq 11.1 \mathrm{mmol} / \mathrm{l}$ (casual is defined as any time of day without regard to the time passed since the last meal), or fasting plasma glucose $\geq 7.0 \mathrm{mmol} / \mathrm{l}$ (fasting is defined as no caloric intake for at least 8 hours), or two-hour glucose $\geq 11.1 \mathrm{mmol} / \mathrm{l}$ during an oral glucose tolerance test (OGTT), detection of islet cell autoantibodies (ICA), glutamic acid decarboxylase 65 (GAD-65) autoantibodies, and insulinoma-associated protein-2 (IA-2), and the presence or absence of symptoms defined by the International Society for Paediatric and Adolescent Diabetes (ISPAD) Clinical Practice Consensus
Guidelines 2014 Compendium [15]. The control group $(n=40)$ was comprised of healthy children who attended a standard systematic examination in the paediatric practice. For the purpose of this study the following tests were performed to rule out underlying diseases in the control group. DM was excluded in the control group based on blood glucose measurements (fasting plasma glucose $<7.0 \mathrm{mmol} / \mathrm{l}$ ) and the absence of DM symptoms. Acute infections were excluded by complete physical examination and normal laboratory values of WBC and CRP. In the initial phase of the study, autoimmune diseases were excluded by negative personal and family history, the absence of clinical symptoms associated with autoimmune diseases, negative or low titre of anti-thyroid peroxidase (TPO) and thyroglobulin and negative celiac disease antibody tests as negative islet cell autoantibodies (ICA), glutamic acid decarboxylase 65 (GAD-65) autoantibodies, and insulinoma-associated protein-2 (IA-2). Informed consent was obtained from parents or legal guardians of all children enrolled in the study. The study was performed in accordance with the Declaration of Helsinki and was approved by the Ethics Committee of Osijek University Hospital and the Faculty of Medicine, University of Osijek.

\section{Methods}

Blood samples were collected in two tubes. Clot activator tubes (Becton, Dickinson, and Company, Franklin Lakes, NJ, USA) were used for CRP, glucose, HMGB1, and autoantibody against $\beta$ cell determination. Samples were centrifuged for 10 minutes at $1500 \mathrm{~g}$. $\mathrm{K}_{2}$ EDTA tubes (Becton, Dickinson, and Company, Franklin Lakes, NJ, USA) were used for WBC count and haemoglobin $A_{1 c}$ $\left(\mathrm{HbA}_{1 \mathrm{c}}\right)$ measurement. Blood samples were collected in the fasting state according to the recommendations of the Clinical Laboratory Standard Institute (CLSI) between 7:00 and 9:00 a.m. [16]. All tests were performed immediately, except those for HMGB1 and autoantibodies against $\beta$ cells. For the determination of HMGB1, serum samples were collected within the first 24 hours of the diagnosis of T1DM and were aliquoted and stored at $-80^{\circ} \mathrm{C}$. The measurements were performed within three months of the sampling. For determination of islet cell autoantibodies (ICA), glutamic acid decarboxylase 65 (GAD-65) autoantibodies and insulinoma-associated protein-2 (IA-2) aliquoted serum samples were stored at $-20^{\circ} \mathrm{C}$ overnight and the analyses were performed the following day. CRP was measured by immunoturbidimetric assay (Roche Diagnostics $\mathrm{GmbH}$, Mannheim, Germany), and glucose was measured by enzymatic method (Beckman Coulter Inc., Brea, USA), both using a Beckman Coulter AU480 analyser (Beckman Coulter Inc., Brea, USA). WBC count was determined using a Sysmex SF-3000 automated haematology analyser (Sysmex Corporation, Kobe, Japan) while HbA1c was determined by the immunoturbidimetric method on a Dimension EXL with an LM analyser (Siemens Healthcare Diagnostics Inc., 
Newark, USA). Determinations of GAD-65 and IA-2 antibodies were performed manually by commercial ELISA kits (EUROIMMUN, Germany) on STAT FAX 2100 microplate reader (Awareness Technology Inc., Palm City, FL, USA). The cut-off for positive results in Croatia is $5 \mathrm{kU} / 1$ for GAD-65 and $10 \mathrm{kU} / \mathrm{L}$ for IA-2 antibodies following the international harmonisation programme (DASP; Diabetes Antibody Standardisation Program). Detection of ICA autoantibodies was done by indirect immunofluorescence assay on frozen sections of blood type 0 human pancreas using indirect immunofluorescence method. ICA values of $\geq 10$ Juvenile Diabetes Foundation (JDF) units were considered positive. These autoantibodies were measured in the ISO 15189:2006 accredited Medical Laboratory, Institute of Clinical Chemistry, University Hospital "Merkur", Zagreb, Croatia. The quality of the performance of the method was validated through the participation in Immunology of Diabetes workshops and proficiency testing programmes of the University of Florida (Gainesville, FL), where these assays achieved $95 \%$ sensitivity, specificity, consistency, and validity. Finally, enzyme-linked immunosorbent assay (ELISA) was used for manual determination of HMGB1 on a Labsystems iEMS Reader MF analyser (Labsystems Oy, Helsinki, Finland) as prescribed. The manufacturer specifies that the assay has an intra- and inter-assay coefficient of variation $(\mathrm{CV})$ of less than $10 \%$. Sample stability was confirmed using five samples, which were retested after storage at $-80^{\circ} \mathrm{C}$ for three months. The determined $\mathrm{CV}$ was less than $6 \%$. BMI was calculated by dividing weight in kilograms by height measured in metres on medical scale and performed by a trained nurse. During measurements each child was in a standing position, in light clothes, and barefoot.

\section{Statistical analysis}

Categorical data are represented by absolute and relative frequencies. The numerical data are described by the median and the limits of the interquartile range. Variations of category variables were tested by Fisher's exact test. Normality of the distribution of numeric variables was tested by the Shapiro-Wilk test. Differences in numeric variables between the two independent groups were tested by Mann-Whitney $U$ test. The correlation rating is given by Spearman's coefficient of correlation Rho. All $p$ values were two-sided. The level of significance was set to alpha $(\alpha)=0.05$. For statistical analysis, the statistical program MedCalc Statistical Software version 17.8.2 was used (MedCalc Software bvba, Ostend, Belgium; http://www. medcalc.org; 2017).

\section{Results}

Our case-control study included 136 children, including $96(70.5 \%)$ with newly diagnosed T1DM and 40 $(29.5 \%)$ controls - healthy children, without T1DM, autoimmune, or any acute infectious or inflammatory diseases.
Table 1. Demographic and clinical characteristics of study groups $(N=136)$

\begin{tabular}{|c|c|c|c|}
\hline \multirow[t]{2}{*}{ Factor } & \multicolumn{2}{|c|}{ Median (interquartile range) } & \multirow[t]{2}{*}{$p$-value } \\
\hline & $\begin{array}{c}\text { T1DM } \\
\text { group } 1^{*} \\
n=96\end{array}$ & $\begin{array}{l}\text { Control } \\
\text { group } \\
n=40\end{array}$ & \\
\hline Age (years) & $12(8-14)$ & $13(13-17)$ & 0.09 \\
\hline BMI $\left(\mathrm{kg} / \mathrm{m}^{2}\right)$ & $17.1(14.9-21.3)$ & $16.9(15.1-18.1)$ & 0.11 \\
\hline $\mathrm{HbA}_{1 \mathrm{c}}(\%)$ & $7.9(7.2-8.6)$ & $5.1(4.7-5.5)$ & 0.03 \\
\hline HMGB1 $(\mu \mathrm{g} / \mathrm{l})$ & $8.7(5.0-9.8)$ & $1(0.6-1.4)$ & $<0.001$ \\
\hline $\mathrm{CRP}(\mu \mathrm{g} / \mathrm{l})$ & $0.8(0.5-1.3)$ & $0.7(0.2-1.2)$ & 0.12 \\
\hline WBC $\left(10^{9}\right.$ cells/ 1$)$ & $6.1(4.9-7.1)$ & $7(5.9-8.1)$ & 0.05 \\
\hline
\end{tabular}

BMI-body mass index, $H b A_{I c}$ - haemoglobin $A_{l c}, H M G B I$ - high-mobility group box 1 protein, $C R P-C$-reactive protein, $W B C-$ white blood cells, ${ }^{*} T 1 D M-$ type 1 diabetes mellitus, group 1 - children with TIDM, ${ }^{\dagger}$ Mann-Whitney $U$ test

The demographic and clinical characteristics are described in Table 1.

There were no significant differences in age $(p=0.09)$ and BMI values $(p=0.11)$ between group 1 and the control group. Group 1 diabetics had significantly higher levels of glycated haemoglobin $\left(\mathrm{HbA}_{1 \mathrm{c}}\right)$ compared to the control group ( $p=0.03$ ) (Table 1). In comparison to the control group, subjects with diabetes had significantly higher levels of HMGB1 $(p<0.001)$; however, the levels of CRP and WBC were not different (Table 1).

Nonetheless, no correlation was found between HMGB1 and $\mathrm{HbA}_{1 \mathrm{c}}$ in group 1 with T1DM (Spearman's coefficient of rank correlation: rho $=-0.081 ; p=0.44 ; 95 \%$ CI: -0.276-0.122). Similarly, no correlation was found between HMGB1 and BMI (Spearman's coefficient of rank correlation: $r h o=0.073 ; p=0.48 ; 95 \% \mathrm{CI}:-0.130-0.269$ ). To assess the intensity of the autoimmune response against pancreatic $\beta$ cells, we measured the serum level of autoantibodies. Complete measurements of GADA-65, IA-2, and ICA were available for the 96 children with T1DM. Of the 96 children with T1DM, $58.3 \%$ were GADA, $44.8 \%$ were IA-2 positive, and $82.9 \%$ were positive for ICA. A statistically higher percentage of positive children for GADA, IA-2, and ICA antibodies were present in group 1 compared to the control group $(p \leq 0.001)$ (Table 2$)$.

In Group $1(n=96)$, we also tested serum levels of HMGB 1 and the presence of autoantibodies, and none of those antibodies correlated with the level of HMGB1 (Table 3).

\section{Discussion}

In our case-control study children with T1DM had one or more $\beta$-cell-specific autoantibodies in their serum. All of the tested antibodies (GAD-65, IA-2, ICA) were, as expected, significantly more positive in children with T1DM compared to the control group with healthy children, in 
Table 2. Distribution of children according to the presence of autoantibodies in studied groups $(N=136)$

\begin{tabular}{|c|c|c|c|c|}
\hline \multirow{2}{*}{$\begin{array}{l}\text { Autoanti- } \\
\text { bodies }\end{array}$} & \multicolumn{3}{|c|}{ Number $(\%)$ of children } & \multirow[t]{2}{*}{$p$-value } \\
\hline & $\begin{array}{c}\text { T1DM } \\
\text { group } 1^{*}\end{array}$ & $\begin{array}{c}\text { Control } \\
\text { group }\end{array}$ & Total & \\
\hline \multicolumn{5}{|l|}{ GAD-65 } \\
\hline Negative & $40(41.7)$ & $40(100)$ & $80(58.8)$ & \multirow[t]{2}{*}{$<0.001$} \\
\hline Positive & $56(58.3)$ & $0(0 \%)$ & $56(41.2)$ & \\
\hline \multicolumn{5}{|l|}{ IA-2 } \\
\hline Negative & $53(55.2)$ & $40(100)$ & $93(68.3)$ & \multirow[t]{2}{*}{$<0.001$} \\
\hline Positive & $43(44.8)$ & $0(0 \%)$ & $43(31.7)$ & \\
\hline \multicolumn{5}{|l|}{ ICA } \\
\hline Negative & $17(17.1)$ & $40(100)$ & 57 (41.9) & \multirow[t]{2}{*}{$<0.001$} \\
\hline Positive & $79(82.9)$ & $0(0 \%)$ & $79(58.1)$ & \\
\hline
\end{tabular}

$\overline{\text { GAD-65 - glutamic acid decarboxylase } 65 \text { autoantibodies, IA-2 - insulinoma- }}$ associated protein, ICA - islet cell autoantibodies, ${ }^{*}$ TIDM - type 1 diabetes mellitus, group 1 - children with TIDM, ${ }^{\dagger}$ Fisher's Exact Test

Table 3. Comparison of the presence of autoantibodies with the HMGB1 serum levels in group $1(N=96)$

\begin{tabular}{|c|c|c|c|}
\hline \multirow[t]{2}{*}{$\begin{array}{l}\text { Autoanti- } \\
\text { bodies }\end{array}$} & \multicolumn{2}{|c|}{$\begin{array}{c}\text { Median (interquartile range) } \\
\text { HMGB1 }(\mu \mathrm{g} / \mathrm{l})\end{array}$} & \multirow[t]{2}{*}{$p$-value } \\
\hline & Negative & Positive & \\
\hline GAD-65 & $8.7(6.65-10.1)$ & $8.7(5.3-9.9)$ & 0.61 \\
\hline IA-2 & $8.7(5.3-9.9)$ & $8.7(5.5-10.0)$ & 0.69 \\
\hline ICA & $9(7.7-10.1)$ & $8.6(5.3-9.9)$ & 0.48 \\
\hline
\end{tabular}

$\overline{\text { GAD-65 - glutamic acid decarboxylase } 65 \text { autoantibodies, IA-2 - insulinoma- }}$ associated protein, ICA - islet cell autoantibodies, "Mann-Whitney U test

whom normal (negative) antibody values were expected. However, the level of HMGB1 and the level of antibodies did not correlate.

T1DM is a chronic disease in which genetic predisposition and environmental influences, predominately early in life, induce pancreatic $\beta$-cell autoimmunity, resulting in loss of function and destruction. Once $\beta$-cell autoimmunity is established the progression towards clinical type 1 diabetes may be classified into three stages: (1) asymptomatic $\beta$-cell autoimmunity with normoglycaemia, (2) asymptomatic $\beta$-cell autoimmunity with dysglycaemia, and (3) symptomatic type 1 diabetes mellitus [17]. One or more $\beta$-cell autoantibodies are present in $90 \%$ of children with T1DM. The measured levels of antibodies in our study are similar to those seen in previous studies, where insulin antibodies were expressed first, especially in younger children [18]. This finding highlights that HMGB1 has potential importance as a T1DM inflammation biomarker and therefore supports the possibility of identifying T1DM high-risk individuals and achieving successful prevention by affecting a potential therapeutic target.
This case-control study has some notable limitations, such as the relatively small number of children involved, non-random selection of the study groups, and the comparison of T1DM children only to those without any inflammation. Recent studies have proposed that HMGB1 is a crucial mediator in the pathogenesis of many diseases, such as sepsis, cancer, chronic kidney disease, and autoimmunity diseases like arthritis, vasculitis, and lupus [19, 20]. All of these conditions are linked to inflammation. Given that T1DM is also an inflammatory process, it is reasonable to expect higher concentrations of HMGB1.

Han et al. [10] showed in animal studies that HMGB1 functioned as a potent innate immune mediator contributing to autoimmune progression during T1DM development. These researchers also showed that HMGB1 could be passively released from damaged pancreatic $\beta$ cells or secreted by islet infiltrated auto reactive immune cells. They also found that blocking HMGB1 significantly inhibited insulitis progression and diabetes development in NOD mice. These results may be of interest for developing novel therapeutic strategies to mitigate or prevent T1DM and, to some extent, justify the potential value of its measurement.

Another study by Arrigo et al. [21] demonstrated the important role of HMGB1 in the inflammatory process associated with childhood obesity. They observed extremely high values of HMGB1 in obese children, connected with IL-6, TNF- $\alpha$, IL-18, resistin, and adiponectin and in correlation with BMI. However, our HMGB1 concentrations did not correlate with BMI values. This finding maybe observed because the BMI values in our T1DM children were notably lower than those from previous studies, reducing the weight of inflammation due to obesity in our case. This result also supports the assumption of higher concentrations of HMGB1 caused by T1DM inflammation in our case. Interestingly, no correlation was observed between $\mathrm{HbA}_{1 \mathrm{c}}$ and HMGB1, despite the positive correlation between them observed in some previous studies, albeit on adults with T2DM [22, 23].

HMGB1 protein is relatively specific for autoimmune diseases according to reference [24], which is not the case with other, less specific inflammatory markers like CRP, so it may represent a more advantageous potential marker of disease activity. Additionally, in murine models of diabetes HMGB1 blockade protects against the development of diabetes. This indicates that HMGB1 might be the early driver of pancreatic cell destruction. Our results are consistent with such a possibility but would need vigorous further testing, perhaps using anti-HMGB1-directed treatment in the clinical setting of T1DM prevention.

\section{Implications of the results for clinical practice}

The results of this study can significantly contribute to a better understanding of the pathogenetic mechanisms of T1DM, which provides a basis for the development of new and more effective therapeutic approaches in all stages of treating children with T1DM. 


\section{Conclusions}

In conclusion, our results show significantly higher values of HMGB1 in children with T1DM compared to the control group. To the best of our knowledge, this casecontrol study is the first to demonstrate a relationship between HMGB1 and T1DM in children. Further studies are needed to confirm our preliminary results, to determine the timing of the appearance of HMGB1 in disease development, and to clarify the role of HMGB1 in the pathogenesis of autoimmune initiation in T1DM.

The authors declare no conflict of interest.

\section{References}

1. Kahanovitz L, Sluss PM, Russell SJ (2017): Type 1 diabetes - a clinical perspective. Point Care 16: 37-40.

2. Gong Y, Jin X, Wang QS, et al. (2014): The involvement of high mobility group 1 cytokine and phospholipases A2 in diabetic retinopathy. Lipids Health Dis 13: 156.

3. Rosin DL, Okusa MD (2011): Dangers within: DAMP responses to damage and cell death in kidney disease. J Am Soc Nephrol 22: 416-425.

4. Praasula SP, Sameera K (2013): Trace elements in diabetes mellitus. J Clin Diagn Res 7: 1863-1865.

5. Jiang G, Wang Y, Yun J, et al. (2015): HMGB1 release triggered by the interaction of live retinal cells and uveitogenic $\mathrm{T}$ cells is Fas/FasL activation-dependent. J Neuroinflammation 12: 179.

6. Sims GP, Rowe DC, Rietdijk ST, et al. (2010): HMGB1 and RAGE in inflammation and cancer. Ann Rev Immunol 28: 367-388.

7. El-Asrar AM, Nawaz MI, Kangave D, et al. (2011): Highmobility group box-1 and biomarkers of inflammation in the vitreous from patients with proliferative diabetic retinopathy. Mol Vision 17: 1829-1838.

8. Akbar M, Gilchrist DS, Kitson SM, et al. (2017): Targeting danger molecules in tendinopathy: the HMGB1/TLR4 axis. RMD Open 3: e000456.

9. Zhang S, Zhong J, Yang P, et al. (2009): HMGB1, an innate alarmin, in the pathogenesis of type 1 diabetes. Int J Clin Exp Pathol 3: 24-38.

10. Han J, Zhong J, Wei W, et al. (2008): Extracellular highmobility group box 1 acts as an innate immune mediator to enhance autoimmune progression and diabetes onset in NOD mice. Diabetes 57: 2118-2127.

11. Kanellakis P, Agrotis A, Kyaw TS, et al. (2011): Highmobility group box protein 1 neutralization reduces development of diet induced atherosclerosis in apolipoprotein e-deficient mice. Arteriosclerosis, Thrombosis, and Vascular Biology Journal 31: 313-319.

12. Montes VN, Subramanian S, Goodspeed L, et al. (2015): AntiHMGB1 antibody reduces weight gain in mice fed a high-fat diet. Nutrition \& Diabetes 15: e161.

13. Yu Y, Yang L, Lv J, et al. (2015): The role of high mobility group box 1 (HMGB-1) in the diabetic retinopathy inflammation and apoptosis. Int J Clin Exp Pathol 8: 6807-6813.
14. Wang Y, Zhong J, Zhang X, et al. (2016): The Role of HMGB1 in the Pathogenesis of Type 2 Diabetes. Journal of Diabetes Research: 2543268.

15. Craig ME, Jefferies C, Dabelea D, et al. (2014): Definition, epidemiology, and classification of diabetes in children and adolescents. Pediatric Diabetes 15: 4-17.

16. Clinical and Laboratory Standards Institute (CLSI): Procedure for the Collection of Diagnostic Blood Specimens by Venipuncture. Approved Standard - Fifth Edition. CLSI document H3-A5. Wayne, PA, USA, 2007.

17. Insel RA, Dunne JL, Atkinson MA, et al. (2015): Staging presymptomatic type 1 diabetes: a scientific statement of JDRF, the Endocrine Society, and the American Diabetes Association. Diabetes Care 38: 1964-1974.

18. Awadalla SA, AL-Hakbani M (2017): The predictive value of diabetes-related antibodies in children with type 1 diabetes mellitus and their siblings. International Journal of Diabetes in Developing Countries 37: 248-253.

19. Bruchfeld A, Wendt M, Bratt J, et al. (2011): High-mobility group box-1 protein (HMGB1) is increased in antineutrophilic cytoplasmatic antibody (ANCA)-associated vasculitis with renal manifestations. Molecular Medicine 17: 9-35.

20. Urbonaviciute V, Voll RE (2011): High-mobility group box 1 represents a potential marker of disease activity and novel therapeutic target in systemic lupus erythematosus. J Intern Med 270: 309-318.

21. Arrigo T, Chirico V, Salpietro V, et al. (2013): High-mobility group protein B1: a new biomarker of metabolic syndrome in obese children. Eur J Endocrinol 168: 631-638.

22. Yin J, Jin D, Wang H (2015): Serum glycated albumin is superior to hemoglobin A1c for correlating with HMGB1 in coronary artery disease with type 2 diabetic mellitus patients. Int J Clin Exp Med 8: 4821-4825.

23. Zhao D, Wang Y, Tang K, Xu Y (2013): Increased serum HMGB1 related with HbA1c in coronary artery disease with type 2 diabetes mellitus. Int J Cardiol 168: 1559-1560.

24. Harris HE, Andersson U, Pisetsky DS (2012): HMGB1: a multifunctional alarmin driving autoimmune and inflammatory disease. Nat Rev Rheumatol 8: 195-202. 